\title{
Graft Copolymerization of Butyl Acrylate and 2-Ethyl Hexyl Acrylate from Labile Chlorines of Poly(vinyl chloride) by Atom Transfer Radical Polymerization
}

\author{
NIYAZI BICAK, MESUT OZLEM \\ Department of Chemistry, Istanbul Technical University, Maslak 80626 Istanbul, Turkey
}

Received 19 May 2003; accepted 30 July 2003

\begin{abstract}
Trace amounts of labile chlorines present in poly(vinyl chloride) (PVC) were found to act as initiation sites for the preparation of graft copolymers of PVC by copper-mediated atom transfer radical polymerization (ATRP). High grafting yields were attained during the graft copolymerizations of $n$-butyl acrylate $(161.8 \%)$ and 2-ethyl hexyl acrylate $(51.2 \%)$ in $7.5 \mathrm{~h}$. In both cases, the grafting proceeded with first-order kinetics with respect to the monomer concentrations, this being typical for ATRP. Gel permeation chromatography traces of the resulting products did not exhibit additional peaks attributable to the formation of free homopolymers. The presented procedure offers an efficient means of preparing self-plasticized PVC structures. ( 2003 Wiley Periodicals, Inc. J Polym Sci Part A: Polym Chem 41: 3457-3462, 2003

Keywords: poly(vinyl chloride) (PVC); atom transfer radical polymerization (ATRP); labile chlorine; graft copolymers; butyl acrylate; 2-ethyl hexyl acrylate
\end{abstract}

\section{INTRODUCTION}

Copper-mediated atom transfer radical polymerization (ATRP) has been one of the most efficient controlled/living polymerization methods since its introduction by Wang and Matyjaszewski. ${ }^{1}$ The controlled chain growth and living nature of ATRP make it very useful for the preparation of well-defined block and graft copolymers. ${ }^{2,3}$ Another advantage of this method over common radical initiation methods is that only negligible homopolymer formation is observed when it is employed in graft copolymerizations., ${ }^{4,5}$ For this reason, ATRP is also very efficient for grafting from solid surfaces. Generally homopolymer formation is not observed in this graft method. ${ }^{6-8}$

In this study, graft copolymers of poly(vinyl chloride) (PVC) were prepared by ATRP. The main purpose of the PVC grafting was to impart a plasticizing effect. Mixing with a liquid plasticizer

Correspondence to: N. Bicak (E-mail: bicak@itu.edu.tr) Journal of Polymer Science: Part A: Polymer Chemistry, Vol. 41, 3457-3462 (2003) ๑) 2003 Wiley Periodicals, Inc. and self-plasticization by the copolymerization of vinyl chloride and vinyl acetate are commercially important methods of plasticizing PVC. ${ }^{9}$

The partial modification of PVC by sodium diethyl dithiocarbamate and subsequent heating ${ }^{10}$ or UV irradiation ${ }^{11,12}$ has also been demonstrated to be efficient for grafting acrylate and $N$-vinyl pyrrolidone monomers from PVC.

Graft copolymers of PVC have also been prepared by ATRP from a PVC copolymer possessing vinyl chloroacetate segments (a few percent). ${ }^{13}$ The chloroacetyl groups of the polymer have been used as initiation sites for ATRP because of the inertness of the chlorine atoms of PVC itself for the ATRP initiation. PVC differs from single alkyl halides, in that the chlorine atoms of PVC do not readily undergo substitution reactions under ordinary conditions. Under drastic conditions, dehydrochlorination takes place and yields conjugated polyene structures. However, commercial PVC contains minute amounts (ca. 1\%) of labile chlorine atoms, which are responsible for the initiation of thermal dehydrochlorination. ${ }^{14,15} \mathrm{Al}$ lylic chlorines and tertiary chlorines at branching 
points are considered to be labile chlorines. The number of labile chlorines depends on the polymerization conditions of vinyl chloride. Head-tohead monomer addition and subsequent chain transfer to the monomer may lead to allylic chloride and branch defects. Branching may also result from chain transfer to the monomer after back-biting and chain transfer to PVC. ${ }^{16}$ Labile or active chlorines have been shown to be very useful for cationic graft copolymerization with some vinyl monomers, including isobutylene, with dialkyl aluminum as a catalyst. ${ }^{17}$ The same catalysis mechanism has been employed for quantitative allylation via active chlorines of PVC. ${ }^{18}$ Caraculacu et al. ${ }^{19}$ suggested an analytical procedure for the determination of the labile chlorines in PVC.

This method relies on substitution by the sodium salt of dithiocarbonic acid through the labile chlorines of PVC, and the amount of replaced chlorine is determined from the sulfur content of the resulting product. However, the reliability of this method is doubtful because the labile chlorine content found is not constant and increases (up to $4.0 \%$ ) with the contact times of the reagent.

In this study, we investigated the utility of ATRP in the grafting of $n$-butyl acrylate (BA) and 2-ethyl hexyl acrylate (EHA) by initiation via the labile chlorines of PVC.

More recently, Percec and coworkers ${ }^{20,21}$ succeeded in grafting styrene and butyl methacrylate monomers from labile chlorines of PVC in diphenyl ether at $120^{\circ} \mathrm{C}$. In this study, we studied the graft copolymerization of BA and EHA from labile chlorines of PVC by copper-mediated ATRP with 1,2-dichlorobenzene at $90{ }^{\circ} \mathrm{C}$. Hexylated triethylenetetraamine (H-TETA) was used as a ligand and formed organosoluble copper complexes. This ligand provided entirely homogeneous ATRP conditions. ${ }^{22}$ The kinetics of the copolymerization were investigated, and the structures of the resulting copolymers were confirmed by NMR spectroscopy.

\section{EXPERIMENTAL}

\section{Materials}

BA (Fluka) was purified by distillation. EHA (Aldrich) was rendered inhibitor-free via shaking with a $0.1 \mathrm{M} \mathrm{NaOH}$ solution. PVC [Aldrich; numberaverage molecular weight $\left(M_{\mathrm{n}}\right)=93,000$, polydispersity index $(\mathrm{PDI})=1.718$ ] was used as obtained.

\section{Techniques}

${ }^{1} \mathrm{H}$ NMR spectra were obtained with a Bruker 250-MHz NMR spectrometer, with deuterated dimethyl sulfoxide as a solvent and tetramethylsilane as an internal standard.

Fourier transform infrared (FTIR) spectra were recorded with a Matson 1100 IR spectrometer with cast films of the polymer samples.

Gel permeation chromatography (GPC) traces of the graft copolymer samples were taken with an Agillant 1100 series instrument, which consisted of a pump, a refractive-index detector, and Waters Styragel (HR 4, HR 3, and HR 2) columns. Tetrahydrofuran (THF) was used as the eluent, and the flow rate was $0.3 \mathrm{~mL} / \mathrm{min}$. Before the manipulations, copper residues in the samples were removed by reprecipitation twice in acetic acid/ethanol mixtures (1/1). Thus, about $0.2 \mathrm{~g}$ of the light blue products was dissolved in THF (10 $\mathrm{mL}$ ) and precipitated in $25 \mathrm{~mL}$ of the acetic acid/ ethanol mixtures. This procedure gave copperfree white polymers, which were pure enough to use in NMR and GPC experiments. In this way, traces of copper remaining in the samples were transferred into the solution as acetate salts.

\section{Preparation of $\mathrm{CuBr}$}

$\mathrm{CuBr}$ was freshly prepared according to a procedure described in the literature. ${ }^{23}$

\section{Preparation of the Ligand}

The ligand H-TETA [called hexacis-(1,1,4,7,10,10hexyl,1,4,7,10-tetraazadecane) by IUPAC] was prepared by the condensation of triethylenetetramine with 1-bromo hexane as previously described. ${ }^{21}$

\section{Graft Copolymerization}

The graft copolymers of PVC with BA and EHA were prepared in 1,2-dichlorobenzene as a solvent at $90{ }^{\circ} \mathrm{C}$.

\section{Graft Copolymerization of BA from Labile Chlorine Atoms of PVC}

PVC (6.25 g, $0.1 \mathrm{~mol})$ was dissolved in $100 \mathrm{~mL}$ of 1,2-dichlorobenzene. This solution was transferred into a $250-\mathrm{mL}$ flask equipped with a magnetic stirrer and a reflux condenser. Nitrogen was flushed through the solution for $3 \mathrm{~min}$. Then, 14.3 $\mathrm{mL}(01 \mathrm{~mol})$ of BA, $0.325 \mathrm{~g}(0.001 \mathrm{~mol})$ of H-TETA, and $0.08 \mathrm{~g}(0.001 \mathrm{~mol})$ of $\mathrm{Cu}(\mathrm{I}) \mathrm{Br}$ were added to 


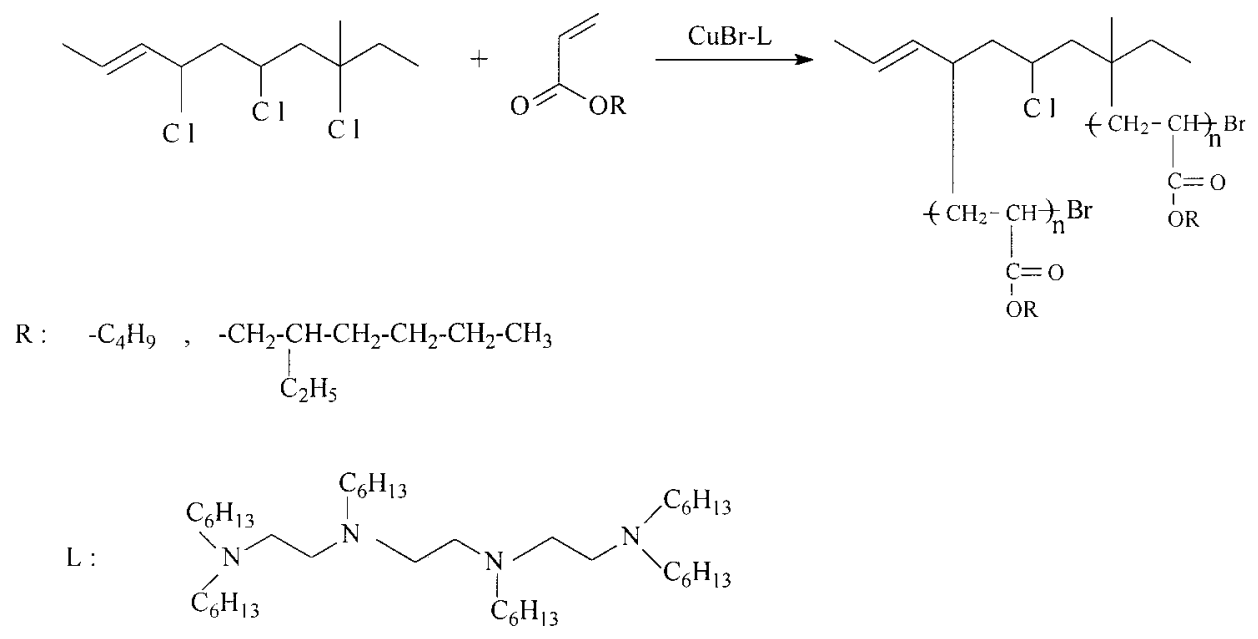

Scheme 1. Grafting of acrylate monomers from labile chlorines of PVC.

the flask. The graft copolymerization was carried out under a nitrogen atmosphere. Then, the flask was immersed in a thermostated oil bath and stirred at $90 \pm 1{ }^{\circ} \mathrm{C}$ at a constant stirring rate (400 rpm). The polymerization was continued for $7.5 \mathrm{~h}$, and the reaction kinetics were followed by the monitoring of the progressive mass increases of the aliquots (ca. $5 \mathrm{~mL}$ of each) taken at predetermined time intervals. These aliquots were poured into THF/acetic acid mixtures $(15 \mathrm{~mL}$, 10:1) to stop the reaction and to remove the copper residues. Then, the polymer solutions were precipitated in ethanol $(40 \mathrm{~mL})$. The products were filtered by suction and washed with ethanol and water. The samples were dried at room temperature for $24 \mathrm{~h}$ in vacuo and weighed.

To separate the free homopolymer from the product, the product was dissolved in THF (10 $\mathrm{mL})$, precipitated in $n$-butanol $(25 \mathrm{~mL})$, and filtered. The filtrate was poured into methanol. However, no precipitate was observed.

\section{Graft Copolymerization of EHA}

The same procedure was followed for the grafting of EHA, except that the aliquots were precipitated in $n$-butanol directly for the removal of the free homopolymer.

\section{RESULTS AND DISCUSSION}

Labile chlorines of PVC resulting from structural defects formed during the radical polymerization of vinyl chloride can act as initiation sites for the direct grafting of PVC by copper-mediated ATRP.
In this way, the graft copolymers PVC-g-poly(EHA) and PVC-g-poly(BA) were prepared with high-conversion yields (Scheme 1).

The graft copolymerizations were carried out in 1,2-dichlorobenzene as a solvent at a constant temperature of $90{ }^{\circ} \mathrm{C}$. 1,2-Dichlorobenzene was selected as the proper solvent for PVC. Other common solvents, such as cyclohexanone and methyl ethyl ketone, were not chosen because of possible side reactions yielding brominated derivatives with cuprous bromide.

The ligand was H-TETA, which formed completely soluble copper complexes in organic solvents. This formulation enabled us to perform ATRP under entirely homogenous conditions.

The graft reaction kinetics were followed by the progressive mass increases of the polymer samples taken from the reaction mixture at different time intervals. The grafting of PVC at $90^{\circ} \mathrm{C}$ was reasonably fast for both monomers. Grafting yields of 161.8 and $51.2 \%$ were attained in $7.5 \mathrm{~h}$ for BA and EHA monomers, respectively (Table 1).

Interestingly, no free homopolymers of BA and EHA were detected throughout the polymerizations. For the detection of any probable free homopolymer formation, the graft copolymer samples were precipitated in butanol, and butanol filtrates were added to an excess of methanol. No precipitate was observed. These observations clearly indicated that the homopolymers were not present in the graft products.

Moreover, only one peak appeared in each GPC trace (Fig. 1), and this revealed that neither unreacted PVC nor acrylate homopolymers were present in the reaction products. In the case of 
Table 1. Graft Copolymerization Characteristics of BA and EHA from PVC

\begin{tabular}{lccccc}
\hline & $\begin{array}{c}\text { Initial Concentration } \\
(\mathrm{M})^{\mathrm{a}}\end{array}$ & $\begin{array}{c}\text { Graft Yield } \\
(\%)^{\mathrm{c}}\end{array}$ & $\begin{array}{c}\text { First-Order } \\
\text { Rate Constant }\end{array}$ & $M_{\mathrm{n}} \times 10^{-3}$ & $\mathrm{PDI}^{\mathrm{d}}$ \\
\hline BA & 1 & $100 / 1$ & 161.8 & $3.34 \times 10^{-5} \mathrm{~s}^{-1}$ & 130 \\
EHA & 1 & $100 / 1$ & 51.2 & $4.48 \times 10^{-6} \mathrm{~s}^{-1}$ & 106 \\
\hline
\end{tabular}

${ }^{\text {a }}$ In 1,2-dichlorobenzene at $90 \pm 1{ }^{\circ} \mathrm{C}$.

${ }^{\mathrm{b}}$ Molar ratio of the monomer to the copper complex.

${ }^{\mathrm{c}}$ Calculated from the mass increase for a reaction time of $7.5 \mathrm{~h}$.

${ }^{\mathrm{d}}$ For the graft copolymer.

$161.8 \%$ grafting, the molar ratio of BA units to the vinyl chloride repeat units would be (if we ignore minor changes in mass differences arising from replaced chlorines at the initiation sites and added halogens at the chain ends) as follows:

$$
\frac{161.8 / 128}{100 / 62.5}=0.79
$$

where 128 and 62.5 are the molecular weights of BA and vinyl chloride, respectively. In other words, there are 79 BA repeat units per 100 repeat units of PVC.

Similarly, the estimation for $51.2 \%$ grafting with EHA yielded 0.174 EHA units per repeating unit of PVC.

Almost linear plots of $\ln \left(M_{0} / M\right)$ versus time were obtained for BA and EHA graft reactions (Fig. 2; $M_{0}$ is the initial monomer concentration and $M$ is the monomer concentration at any time, which is found by the subtraction of the amounts of the monomers involved in grafting from the initial monomer concentrations). The linearity of the semilogarithmic plots implied first-order kinetics of grafting for both monomers.

The corresponding rate constants were 3.34 $\times 10^{-5}$ and $4.48 \times 10^{-6} \mathrm{~s}^{-1}$ for BA and EHA, respectively. The low grafting rate for EHA might be due to the high molecular weight of this monomer. Moreover, the $M_{\mathrm{n}}$-conversion plots were also linear (Fig. 3) this being typical behavior for copper-mediated ATRP. Because the PDI of the commercial PVC that we used was high (1.718), the PDIs of the graft copolymer samples were relatively high and typically were $1.99-1.56$, as shown in Figure 4. Sharp increases were observed in the PDIs at the beginning of the reactions. These values were around 1.6 at high conversions.

The presence of attached chains of $\mathrm{BA}$ and EHA was also evidenced by FTIR and ${ }^{1} \mathrm{H}$ NMR spectra of the graft products. In the FTIR spectra, the appearance of characteristic $\mathrm{C}=\mathrm{O}$ stretching vibrations of the acrylate repeat units at $1715 \mathrm{~cm}^{-1}$ established the graft structures.

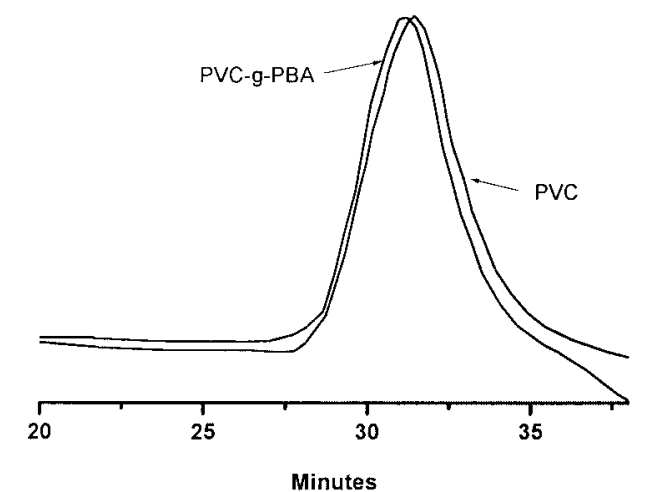

(a)

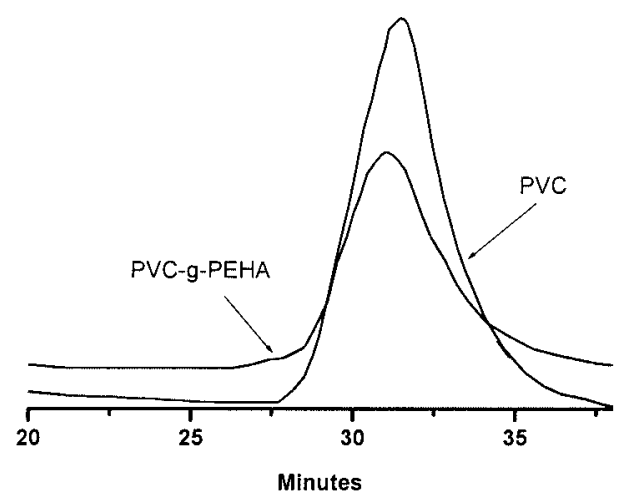

(b)

Figure 1. GPC traces of graft copolymers of $\mathrm{PVC}$ obtained in a reaction time of $7.5 \mathrm{~h}$ : (a) PVC-g-poly(BA) and (b) PVC-g-poly(EHA). 


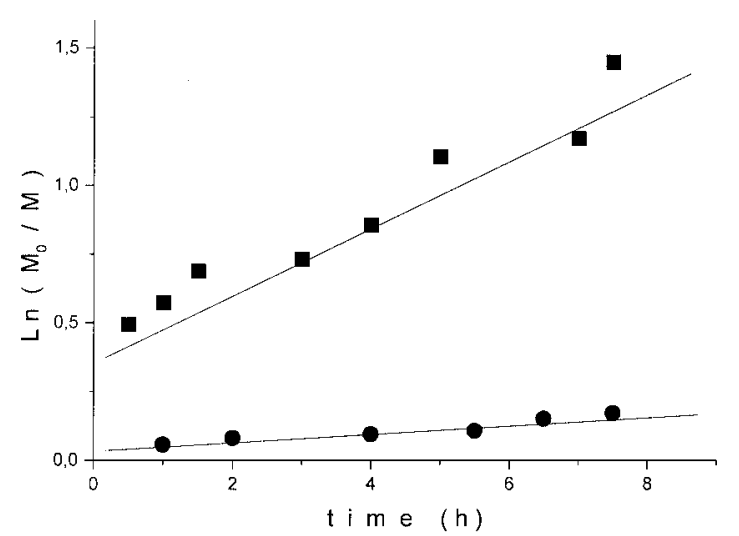

Figure 2. First-order kinetic plots for the graft copolymerization of (ם) BA and (- EHA at $90{ }^{\circ} \mathrm{C}(\mathrm{CuBr}-\mathrm{L} /$ $[\mathrm{M}]=0.0018)$.

The ${ }^{1} \mathrm{H}$ NMR patterns (Fig. 5). of the graft copolymers seemed to be superpositioned ${ }^{1} \mathrm{H}$ NMR spectra of the individual components. Typical signals of the methyl protons of the butyl or hexyl groups were discernible as broad bands around $1 \mathrm{ppm}$, which indicated the incorporation of BA and EHA chains into the graft products. All the other proton signals were broadened at 1.5-3 and 4-4.9 ppm. The former signal must be associated with the sum of the methine protons of PVC with the $-\mathrm{OCH}_{2}$ - protons of the ester groups of the acrylate units. The integral ratio of the signal at 4-4.9 ppm to the signal at $1 \mathrm{ppm}$ yielded $0.75 \mathrm{BA}$ repeat units per PVC repeat unit. This rough estimation was very close to the value (0.79) obtained from the mass increase.

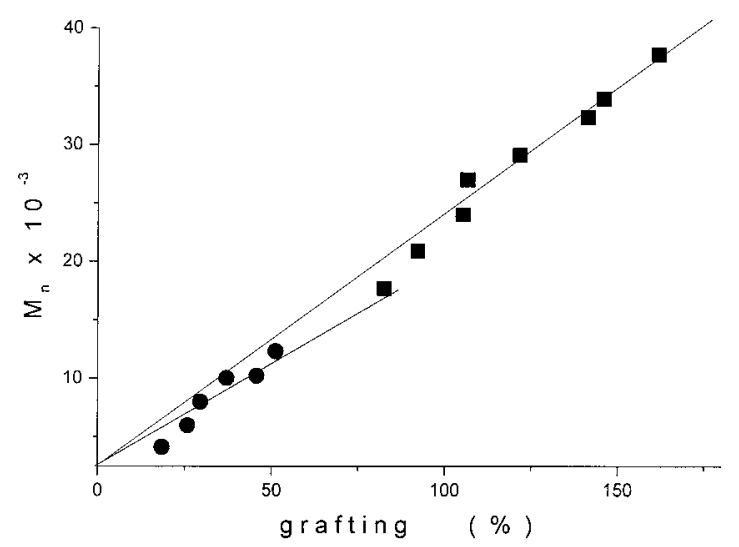

Figure 3. Total molecular weights of the graft chains accumulated on PVC versus the conversion for the grafting of $(\square) \mathrm{BA}$ and $(\mathbf{}) \mathrm{EHA}$ at $90^{\circ} \mathrm{C}(\mathrm{CuBr}-\mathrm{L} /[\mathrm{M}]=$ 0.0018).

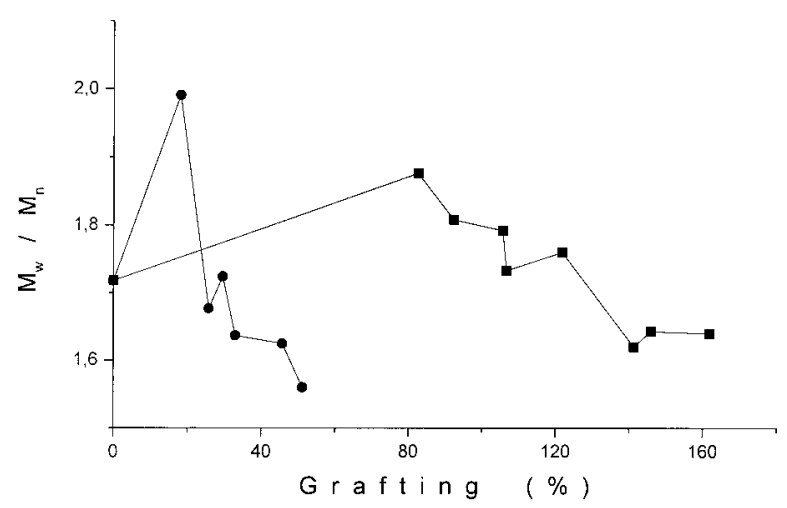

Figure 4. PDI $\left(M_{\mathrm{w}} / M_{\mathrm{n}}\right)$ as a function of the grafting yield for (ם) BA and (- $\mathrm{EHA}$ at $90{ }^{\circ} \mathrm{C}\left(M_{\mathrm{w}} / M_{\mathrm{n}}\right.$ of starting $\mathrm{PVC}=1.718)$.

A similar inspection of an EHA graft sample obtained after $7.5 \mathrm{~h}$ yielded 14.3 EHA repeat units per 100 repeat units of PVC. This amount was also comparable to the value (17.4) found from the mass increase of the sample. As a result, the spectroscopic measurements qualitatively confirmed the expected graft structures.

We also attempted to estimate the lengths of the polyacrylate chains by the determination of the labile chlorine content of the starting PVC with the method suggested by Caraculacu et al. ${ }^{19}$ However, the values that we found were in the range of $0-2.6 \%(\mathrm{~mol} / \mathrm{mol})$ and varied with both the temperature and contact time of the sulfur reagent. After $7.5 \mathrm{~h}$ at $90{ }^{\circ} \mathrm{C}$, the determined value $(2.6 \%)$ corresponded to 2.6 initiation sites per 100 repeat units of PVC. The ratio of the BA repeat units to the number of initiation sites was, therefore, 79/2.6 $=30.4$. In other words, an average of 30 repeat units of BA might be present in

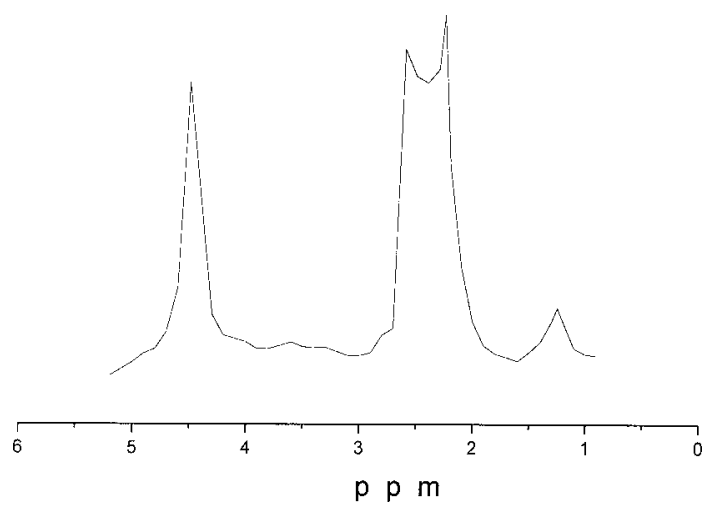

Figure 5. ${ }^{1} \mathrm{H}$ NMR spectrum of PVC-g-poly(BA) obtained after a reaction time of $7.5 \mathrm{~h}$. 
each graft chain. However, the reliability of this value (2.6) is doubtful. We believe that this assumption is not true not only because of the unreliability of the labile chlorine content found but also because of the reactivity differences of the halogens at the ends of the graft chains and the labile chlorines in the backbone of PVC.

Prolonged graft reactions $(>24 \mathrm{~h})$ resulted in gelation. This might be largely due to the formation of additional new primary radicals during the chain growth. The combination of these radicals was expected to form partially crosslinked gel products with longer reaction times.

\section{CONCLUSIONS}

BA and EHA can be efficiently grafted from labile chlorines of commercial PVC by copper-mediated ATRP. This process does not require the use of additional comonomers or modified functional groups with active halides for the initiation of ATRP. The presented process offers a relatively simple method of efficiently grafting BA and EHA for the preparation of self-plasticized PVC.

The Istanbul Technical University Institute of Science and Technology is acknowledged for its financial support.

\section{REFERENCES AND NOTES}

1. Wang, J. S.; Matyjaszewski, K. Macromolecules 1995, 28, 7901.

2. Shipp, D. A.; Wang, J. L.; Matjaszewski, K. Macromolecules 1998, 31, 8005.

3. Liu, S.; Sen, A. Macromolecules 2000, 33, 5106.
4. Grubbs, R. B.; Hawker, C. J.; Dao, J.; Frechet, J. M. Angew Chem Int Ed Engl 1997, 36, 270.

5. Von Werne, T.; Patten, T. E. J Am Chem Soc 1999, 121, 7409.

6. Senkal, F.; Bicak, N. Eur Polym J 2003, 39, 327.

7. Ayres, N.; Haddleton, D. M.; Shooter, A. J.; Pears, D. A. Macromolecules 2002, 35, 3849.

8. Sedjo, R. A.; Mirous, B. K.; Brittain, W. J. Macromolecules 2000, 33, 1492.

9. Marvel, G. S.; Sample, J. H.; Roy, M. F. J Am Chem Soc 1993, 51, 3241.

10. Okawara, M.; Marishita, K.; Emoto, E. Kogyo Kagaku Zasshi 1966, 69, 761.

11. Miyama, H.; Fujii, N.; Shimazaki, Y.; Ikede, K. Polym Photochem 1983, 3, 445.

12. Miyama, H.; Harumiya, N.; Mori, Y.; Tanzave, H. J Biomed Mater Res 1977, 11, 251.

13. Matyjaszewski, K.; Gaynor, S. G.; Paik, H. J Macromol Rapid Commun 1998, 19, 47.

14. Bengough, W. I.; Onozuka, M. Polymer 1965, 6, 625.

15. Gressier, J.-C.; Levesque, G.; Bonnans-Plaisance, C. Macromol Chem Rapid Commun 1983, 4, 387.

16. Asandei, A. D.; Percec, V. J Polym Sci Part A: Polym Chem 2001, 39, 3392.

17. Kennedy, J. P. J Polym Sci Part A: Polym Chem 2001, 39, 1675.

18. Pi, Z.; Kennedy, J. P. J Polym Sci Part A: Polym Chem 2001, 39, 307.

19. Caraculacu, A. A.; Bezdadea, E. C.; Istrate, G. J Polym Sci Part A: Polym Chem 1970, 8, 1239.

20. Percec, V.; Cappotto, A.; Barboiu, B. Macromol Chem Phys 2002, 203, 1674.

21. Percec, V.; Asgarzadeh, F. J Polym Sci Part A: Polym Chem 2001, 39, 1120.

22. Acar, M. H.; Bicak, N. J Polym Sci Part A: Polym Chem 2003, 41, 1677.

23. Vogel, A. I. Textbook of Practical Organic Chemistry, 5th ed.; Prentice Hall: London, 1989; p 428. 